\title{
ONE-DIMENSIONAL TUNNEL-JUNCTION FORMULA FOR SCHRÖDINGER PARTICLE
}

\author{
MASAO HIROKAWA* AND TAKUYA KOSAKA ${ }^{\dagger}$
}

\begin{abstract}
We handle all the self-adjoint extensions of the minimal Schrödinger operator for the non-relativistic electron living in the one-dimensional configuration space with a junction. We are interested in every boundary condition corresponding to the individual self-adjoint extension. Thus, we clarify all the types of those boundary conditions of the wave functions of the non-relativistic electron. We find a tunnel-junction formula for the non-relativistic electron passing through the junction. Using this tunnel-junction formula, we propose a mathematical possibility of a tunneljunction device for qubit.
\end{abstract}

Key words. Schrödinger operator, self-adjoint extension, tunnel-junction device, qubit

AMS subject classifications. 47B25, 81P45, 81P68, 81Q10

1. Introduction. In this paper we consider a single electron living in the onedimensional configuration space with a junction. Thus, we consider it the Schrödinger particle. We mathematically simplify our physical set-up in the following: We regard the one-dimensional configuration space with the junction as the space $\Omega_{\Lambda}:=\mathbb{R} \backslash$ $[-\Lambda,+\Lambda]$. The segment $[-\Lambda,+\Lambda]$ is the junction then. We have been interested in the boundary condition of the wave functions of the non-relativistic electron so that the electron's energy operator (i.e., the Schrödinger operator) becomes an observable (i.e., self-adjoint). We have considered some self-adjoint extensions of the minimal Schrödinger operator $H_{0}$ for this configuration space in 2, 4 from the point of the view of a quantum device. We then showed that there are some cases where the wave functions on which a self-adjoint extension acts have their own phase factor at the boundary, $\pm \Lambda$, when the wave functions pass through the junction. On the other hand, we showed that the wave functions do not have such a phase factor when they respectively stay in the left island $\Omega_{\Lambda, L}:=(-\infty,-\Lambda)$ and in the right island $\Omega_{\Lambda, R}:=$ $(+\Lambda,+\infty)$, namely, when there is no exchange between the wave functions living individual islands. Actually, all the self-adjoint extensions of our minimal Schrödinger operator $H_{0}$ can be parameterized by $\left(u_{j k}\right)_{j, k=1,2} \in U(2)$ following the von Neumann's theory. Here, $U(2)$ is the unitary group of the degree 2. In [2] the appearance of the phase factor was shown only in the cases where diagonal entries of the unitary matrix are all zero (i.e., $u_{11}=u_{22}=0$ ). In this paper, we complete the result in [2]. As asserted in Theorem 2.8 below, we will characterize the boundary conditions of all the self-adjoint extensions of the minimal Schrödinger operator $H_{0}$ by just two types of boundary conditions proposed in 2. More precisely, we will actually construct the boundary condition from every matrix of $U(2)$. We will then give a tunnel-junction formula concerning the phase factor for the non-relativistic electron as the Schrödinger particle. We will propose a mathematical idea to make a qubit from a Schrödinger particle through the tunnel-junction formula by controlling the phase factor, even though the spin of the Schrödinger particle cannot be used. In the case where the electron spin should be considered, we studied similar problem for a single relativistic electron as the Dirac particle [3].

\footnotetext{
*Department of Mathematics, Okayama University, Okayama, 700-8530, Japan (hirokawa@math.okayama-u.ac.jp).

${ }^{\dagger}$ Department of Mathematics, Okayama University, Okayama, 700-8530, Japan.
} 
2. Main Theorem. First up, we prepare some mathematical tools and recall some results to state our main theorem, Theorem 2.8.

We respectively define function spaces $A C^{2}\left(\Omega_{\Lambda}\right)$ and $A C_{0}^{2}\left(\Omega_{\Lambda}\right)$ for our configuration space $\Omega_{\Lambda}$ by

$$
\begin{aligned}
A C^{2}\left(\overline{\Omega_{\Lambda}}\right):=\left\{f \in L^{2}\left(\Omega_{\Lambda}\right) \mid\right. & f \text { and } f^{\prime} \text { are absolutely continuous on } \overline{\Omega_{\Lambda}}, \\
& \text { and } \left.f^{\prime}, f^{\prime \prime} \in L^{2}\left(\Omega_{\Lambda}\right)\right\},
\end{aligned}
$$

and

$$
A C_{0}^{2}\left(\overline{\Omega_{\Lambda}}\right):=\left\{f \in A C^{2}\left(\overline{\Omega_{\Lambda}}\right) \mid f=f^{\prime}=0 \text { on } \partial \Omega_{\Lambda}\right\} .
$$

Here $L^{2}\left(\Omega_{\Lambda}\right)$ is the set of all the square Lebesgue-integrable functions on $\Omega_{\Lambda}$, and $\partial \Omega_{\Lambda}$ denotes the boundary $\{-\infty,-\Lambda,+\Lambda,+\infty\}$ of $\Omega_{\Lambda}$. Thus, $f \in A C_{0}^{2}\left(\overline{\Omega_{\Lambda}}\right)$ satisfies the boundary condition: $\lim _{x \rightarrow a} f^{(n)}(x)=0$ for $a \in \partial \Omega_{\Lambda}$ and $n=0,1$.

We denote by $D(A)$ the domain of a linear operator $A$ throughout this paper. Now we introduce our starting object:

Definition 2.1. (Minimal Schrödinger Operator): The 1-dimensional Schrödinger operator $H_{0}$ is defined by

$$
\left\{\begin{array}{l}
D\left(H_{0}\right):=A C_{0}^{2}\left(\overline{\Omega_{\Lambda}}\right), \\
H_{0}:=-\frac{d^{2}}{d x^{2}} .
\end{array}\right.
$$

We call the operator $H_{0}$ the minimal Schrödinger operator.

Following [6, Theorems 8.25(b) and 8.22] respectively, we realize that the minimal Schrödinger operator $H_{0}$ is closed symmetric, and its adjoint operator $H_{0}^{*}$ is given as

$$
\left\{\begin{array}{l}
D\left(H_{0}^{*}\right)=A C^{2}\left(\overline{\Omega_{\Lambda}}\right), \\
H_{0}^{*}=-\frac{d^{2}}{d x^{2}} .
\end{array}\right.
$$

Then, the operation of $H_{0}^{*}$ is the same as that of $H_{0}$ though their domains are different from each other. So, we call the adjoint operator $H_{0}^{*}$ the maximal Schrödinger operator, and moreover, we employ the following naming:

Definition 2.2. For every subspace $\mathcal{D}$ with the condition, $D\left(H_{0}\right) \subset \mathcal{D} \subset D\left(H_{0}^{*}\right)$, we call the restriction, $H_{0}^{*}\left\lceil\mathcal{D}\right.$, of $H_{0}^{*}$ on $\mathcal{D}$ the Schrödinger operator.

We will investigate every boundary condition of the wave functions on which individual self-adjoint extension acts.

It was proved in 2] that both deficiency indices are 2: $n_{+}\left(H_{0}\right)=n_{-}\left(H_{0}\right)=2$, where the deficiency index $n_{ \pm}\left(H_{0}\right)$ is defined as the dimension of the individual deficiency subspace $\mathcal{K}_{ \pm}\left(H_{0}\right):=\operatorname{ker}\left( \pm i-H_{0}^{*}\right): n_{ \pm}\left(H_{0}\right):=\operatorname{dim} \mathcal{K}_{ \pm}\left(H_{0}\right)$. Thus, the minimal Schrödinger operator has uncountably many self-adjoint extensions. More precisely, following the general theory of differential equation and solving simple differential equations: $H_{0}^{*} \psi= \pm i \psi$, we can obtain orthonormal bases, $\left\{L_{ \pm}, R_{ \pm}\right\}$, of the 
deficiency subspaces $\mathcal{K}_{ \pm}\left(H_{0}\right)$, respectively:

$$
\begin{aligned}
L_{+}(x) & :=\left\{\begin{array}{cl}
N e^{(1-i) x / \sqrt{2}} & \text { if }-\infty<x<\Lambda, \\
0 & \text { if } \Lambda<x<\infty,
\end{array}\right. \\
L_{-}(x) & :=\left\{\begin{array}{cl}
N e^{(1+i) x / \sqrt{2}} & \text { if }-\infty<x<\Lambda, \\
0 & \text { if } \Lambda<x<\infty,
\end{array}\right.
\end{aligned}
$$

and

$$
\begin{aligned}
& R_{+}(x):=\left\{\begin{array}{cl}
0 & \text { if }-\infty<x<\Lambda, \\
N e^{(-1+i) x / \sqrt{2}} & \text { if } \Lambda<x<\infty,
\end{array}\right. \\
& R_{-}(x):=\left\{\begin{array}{cl}
0 & \text { if }-\infty<x<\Lambda, \\
N e^{(-1-i) x / \sqrt{2}} & \text { if } \Lambda<x<\infty,
\end{array}\right.
\end{aligned}
$$

with the normalization factor $N=\sqrt[4]{2} e^{\Lambda / \sqrt{2}}$ so that $H_{0}^{*} R_{ \pm}= \pm i R_{ \pm}$and $H_{0}^{*} L_{ \pm}=$ $\pm i L_{ \pm}$. The uniqueness of the differential equations tells us that the individual bases are complete and $n_{+}\left(H_{0}\right)=n_{-}\left(H_{0}\right)=2$. We here note the following relations to use later:

$$
R_{ \pm}^{\prime}(x)=\frac{-1 \pm i}{\sqrt{2}} R_{ \pm}(x) \quad \text { and } \quad L_{ \pm}^{\prime}(x)=\frac{1 \mp i}{\sqrt{2}} L_{ \pm}(x)
$$

and

$$
\left\{\begin{array}{l}
L_{+}(-\Lambda)=R_{+}(+\Lambda)=N e^{(-1+i) \Lambda / \sqrt{2}} \\
L_{-}(-\Lambda)=R_{-}(+\Lambda)=R_{+}(+\Lambda)^{*}=N e^{(-1-i) \Lambda / \sqrt{2}} \\
R_{+}(+\Lambda)^{*}=R_{+}(+\Lambda) e^{-i \sqrt{2} \Lambda}
\end{array}\right.
$$

Following the von Neumann's theory [5, 6], all the self-adjoint extensions $H_{\mathrm{sa}}$ of the minimal Schrödinger operator $H_{0}$ are given as a restriction of the maximal Schrödinger operator $H_{0}^{*}$ on a proper subspace $\mathcal{D}_{\text {sa }}$ with $D\left(H_{0}\right) \subset \mathcal{D}_{\text {sa }} \subset D\left(H_{0}^{*}\right)$ : $H_{\mathrm{sa}}=H_{0}^{*}\left\lceil\mathcal{D}_{\mathrm{sa}}\right.$. Then, von Neumann's theory [5, 6] provides the following proposition:

Proposition 2.3. There is a one-to-one correspondence between self-adjoint extensions $H_{\mathrm{sa}}$ of the minimal Schrödinger operator $H_{0}$ and unitary operators $U$ : $\mathcal{K}_{+}\left(H_{0}\right) \rightarrow \mathcal{K}_{-}\left(H_{0}\right)$ so that the correspondence is given in the following: For every unitary operator $U: \mathcal{K}_{+}\left(H_{0}\right) \rightarrow \mathcal{K}_{-}\left(H_{0}\right)$, the corresponding self-adjoint extension $H_{U}$ is defined by

$$
\left\{\begin{array}{l}
D\left(H_{U}\right):=\left\{\psi=\psi_{0}+\psi^{+}+U \psi^{+} \mid \psi_{0} \in D\left(H_{0}\right), \psi^{+} \in \mathcal{K}_{+}\left(H_{0}\right)\right\}, \\
H_{U}:=H_{0}^{*}\left\lceil D\left(H_{U}\right),\right.
\end{array}\right.
$$

and then its operation is

$$
H_{U}\left(\psi_{0}+\psi^{+}+U \psi^{+}\right)=H_{0} \psi_{0}+i \psi^{+}-i U \psi^{+} .
$$

Conversely, for every self-adjoint extension $H_{\mathrm{sa}}$ of the minimal Schrödinger operator $H_{0}$, there is the corresponding unitary operator $U: \mathcal{K}_{+}\left(H_{0}\right) \rightarrow \mathcal{K}_{-}\left(H_{0}\right)$ so that $H_{\mathrm{sa}}=$ $H_{U}$.

We here introduce some mathematical notation and terminology. We denote by $\overline{\mathbb{R}}$ the set of all extended real numbers: $\overline{\mathbb{R}}:=\mathbb{R} \cup\{+\infty\}$. Since $n_{+}\left(H_{0}\right)=n_{-}\left(H_{0}\right)=2$, 
the deficiency subspaces $\mathcal{K}_{ \pm}\left(H_{0}\right)$ are 2-dimensional Hilbert spaces. We here remember that the sets $\left\{L_{+}, R_{+}\right\}$and $\left\{L_{-}, R_{-}\right\}$are respectively the complete orthonormal systems of the deficiency subspace $\mathcal{K}_{+}\left(H_{0}\right)$ and $\mathcal{K}_{-}\left(H_{0}\right)$. We identify unitary operators $U$ from $\mathcal{K}_{+}\left(H_{0}\right)$ to $\mathcal{K}_{-}\left(H_{0}\right)$ with $2 \times 2$ unitary matrices $\left(u_{j k}\right)_{j, k=1,2}$, making the correspondence by $U: \psi_{j}^{+} \longmapsto \sum_{k=1}^{2} u_{j k} \psi_{k}^{-}, j=1,2$, where $\psi_{1}^{ \pm}:=L_{ \pm}$and $\psi_{2}^{ \pm}:=R_{ \pm}$. So, we often identify the unitary operator $U$ with the unitary matrix $\left(u_{j k}\right)_{j k=1,2}$, and write $U \in U(2)$. We denote by $U(n)$ the unitary group of degree $n \in \mathbb{N}$ throughout this paper. The representation of our $U: \mathcal{K}_{+}\left(H_{0}\right) \rightarrow \mathcal{K}_{-}\left(H_{0}\right)$ in this paper is then:

$$
\left\{\begin{array}{l}
U L_{+}=u_{11} L_{-}+u_{12} R_{-} \\
U R_{+}=u_{21} L_{-}+u_{22} R_{-}
\end{array}\right.
$$

We say that $U$ is diagonal if $u_{j k}=0$ with $j \neq k$. Otherwise, we say $U$ is non-diagonal.

Before stating our main theorem, Theorem 2.8 we recall the two types of selfadjoint extensions found in [2]. We introduce a class of vectors $\alpha=\left(\alpha_{1}, \alpha_{2}, \alpha_{3}, \alpha_{4}\right) \in$ $\mathbb{C}^{4}$ [2, Definition 1]: We say $\alpha \in \mathbb{C}^{4}$ is in a class (Class $\alpha$ ) if and only if

$$
\alpha_{1} \alpha_{4}^{*}-\alpha_{2} \alpha_{3}^{*}=1
$$

and

$$
\alpha_{1} \alpha_{3}^{*}, \quad \alpha_{2} \alpha_{4}^{*} \in \mathbb{R}
$$

For every vector $\alpha$ in the class (Class $\alpha$ ), we give a matrix $B_{\alpha}$ by $B_{\alpha}:=\left(\begin{array}{ll}\alpha_{1} & \alpha_{2} \\ \alpha_{3} & \alpha_{4}\end{array}\right)$, and call it the boundary matrix. We recall that there are at least two types of boundary conditions for self-adjoint extensions of the the minimal Schrödinger operator $H_{0}$ as shown in [2]. We will give a brief outline of its proof in 3.1 .

ThEOREM 2.4. ([2, Theorem 1(ii)ETheorem 2(i)])

(a) For every $\rho=\left(\rho_{+}, \rho_{-}\right) \in \overline{\mathbb{R}}^{2}$, set a subspace $D\left(H_{\rho}\right)$ as

$$
D\left(H_{\rho}\right):=\left\{\psi \in D\left(H_{0}^{*}\right) \mid \psi \text { satisfies the boundary condition BC } B\right\},
$$

where

$(\mathrm{BC} \rho)$

$$
\begin{cases}\rho_{+} \psi(+\Lambda)=\psi^{\prime}(+\Lambda) & \text { if }\left|\rho_{+}\right|<\infty, \\ \psi(+\Lambda)=0 & \text { if } \rho_{+}=\infty, \\ \rho_{-} \psi(-\Lambda)=\psi^{\prime}(-\Lambda) & \text { if }\left|\rho_{-}\right|<\infty, \\ \psi(-\Lambda)=0 & \text { if } \rho_{-}=\infty .\end{cases}
$$

Then, the restriction, $H_{\rho}:=H_{0}^{*}\left\lceil D\left(H_{\rho}\right)\right.$, of the adjoint operator $H_{0}^{*}$ on $D\left(H_{\rho}\right)$ is a self-adjoint extension of the minimal Schrödinger operator $H_{0}$.

(b) For every vector $\alpha$ in the class (Class $\alpha)$, define a subspace $D\left(H_{\alpha}\right)$ by

$$
D\left(H_{\alpha}\right)=\left\{\psi \in D\left(H_{0}^{*}\right) \mid \psi \text { satisfies the boundary condition }(\overline{B C \alpha})\right\},
$$

where

$(\mathrm{BC} \alpha)$

$$
\left(\begin{array}{c}
\psi(+\Lambda) \\
\psi^{\prime}(+\Lambda)
\end{array}\right)=B_{\alpha}\left(\begin{array}{c}
\psi(-\Lambda) \\
\psi^{\prime}(-\Lambda)
\end{array}\right)
$$

Then, the restriction, $H_{\alpha}:=H_{0}^{*}\left\lceil D\left(H_{\alpha}\right)\right.$, of the adjoint operator $H_{0}^{*}$ on $D\left(H_{\alpha}\right)$ is a self-adjoint extension of the minimal Schrödinger operator $H_{0}$. 
We here recall the following lemma:

Lemma 2.5. ([2, Lemma 2]) If $\alpha_{1}, \alpha_{2}, \alpha_{3}, \alpha_{4} \in \mathbb{C}$ in in the class (Class $\alpha$ ), then $\alpha_{j} \alpha_{k}^{*} \in \mathbb{R}$ for each $j, k=1,2,3,4$.

Proof. We only have to show the case where $j \neq j^{\prime}$. Multiplying both sides of (2.9) by $\alpha_{3}^{*}$, we have

$$
\alpha_{3}^{*}=\alpha_{1} \alpha_{3}^{*} \alpha_{4}^{*}-\alpha_{2}^{*}\left|\alpha_{3}\right|^{2} .
$$

Multiply both sides of this equation by $\alpha_{2}$. Then, (2.10) tells us that $\alpha_{2} \alpha_{3}^{*} \in \mathbb{R}$. Combining this fact with (2.9), we have $\alpha_{1} \alpha_{4}^{*}=1+\alpha_{2} \alpha_{3}^{*} \in \mathbb{R}$. Multiplying both sides of (2.9) by $\alpha_{2}$ leads to $\alpha_{2}=\alpha_{1} \alpha_{2} \alpha_{4}^{*}-\left|\alpha_{2}\right|^{2} \alpha_{3}$. Here we used $\alpha_{2}^{*} \alpha_{3}=\alpha_{2} \alpha_{3}^{*} \in \mathbb{R}$. Multiplying both sides of the above representation of $\alpha_{2}$ by $\alpha_{1}^{*}$ brings us to $\alpha_{1}^{*} \alpha_{2} \in \mathbb{R}$. Here we used conditions in (2.10), especially, $\alpha_{1}^{*} \alpha_{3}=\alpha_{1} \alpha_{3}^{*} \in \mathbb{R}$. We reach $\alpha_{3}^{*} \alpha_{4}=$ $\alpha_{1} \alpha_{3}^{*}\left|\alpha_{4}\right|^{2}-\alpha_{2}^{*} \alpha_{4}\left|\alpha_{3}\right|^{2} \in \mathbb{R}$ by multiplying both sides of (2.11) by $\alpha_{4}$ and using the conditions in (2.10). Here we used $\alpha_{2}^{*} \alpha_{4}=\alpha_{2} \alpha_{4}^{*} \in \mathbb{R}$. We have proved our statement for $\alpha_{2} \alpha_{3}^{*}, \alpha_{1} \alpha_{4}^{*}, \alpha_{1}^{*} \alpha_{2}$, and $\alpha_{3}^{*} \alpha_{4}$. It follows from these facts and the conditions in (2.10) that $\alpha_{j} \alpha_{k}^{*} \in \mathbb{R}$ for all the combinations of $j, k=1,2,3,4$.

The following proposition shows how a phase factor appears in the boundary matrices $B_{\alpha}$ with $\alpha \in \mathbb{C}^{4}$ in the class (Class $\alpha$ ):

Proposition 2.6. Let $B_{\alpha}$ be an arbitrary boundary matrix with the vector $\alpha=$ $\left(\alpha_{1}, \alpha_{2}, \alpha_{3}, \alpha_{4}\right) \in \mathbb{C}^{4}$ in the class (Class $\left.\alpha\right)$. Then, one of $\alpha_{1}$ and $\alpha_{2}$ is non-zero at least. So, set $\theta \in[0,2 \pi)$, and $a_{1}, a_{2}, a_{3}, a_{4} \in \mathbb{R}$ as follows: Let $\alpha_{j}$ be $\alpha_{1}$ if $\alpha_{1} \neq 0$, and $\alpha_{2}$ if $\alpha_{1}=0$. Define

$$
\left\{\begin{array}{l}
\theta:=\arg \left(\alpha_{j} /\left|\alpha_{j}\right|\right) ; \\
a_{j}:=\left|\alpha_{j}\right|, \quad a_{k}:=\alpha_{k} \alpha_{j}^{*} /\left|\alpha_{j}\right|, \quad k \neq j .
\end{array}\right.
$$

Then, $B_{\alpha}$ has the following representation:

$$
B_{\alpha}=e^{i \theta}\left(\begin{array}{ll}
a_{1} & a_{2} \\
a_{3} & a_{4}
\end{array}\right) .
$$

Proof. Set $\theta_{j}$ as $\theta_{j}=\arg \alpha_{j}$. Since the vector $\alpha$ is in the class (Class $\alpha$ ), Lemma 2.5 says that $\alpha_{j} \alpha_{k}^{*} \in \mathbb{R}$ for each $j, k=1,2,3,4$. Moreover, (2.9) says that $\alpha_{1} \neq 0$ or $\alpha_{2} \neq 0$. We can rewrite $B_{\alpha}$ as:

$$
B_{\alpha}=\frac{\alpha_{1}}{\left|\alpha_{1}\right|}\left(\begin{array}{cc}
\left|\alpha_{1}\right| & \frac{\alpha_{1}^{*} \alpha_{2}}{\left|\alpha_{1}\right|} \\
\frac{\alpha_{1}^{*} \alpha_{3}}{\left|\alpha_{1}\right|} & \frac{\alpha_{1}^{*} \alpha_{4}}{\left|\alpha_{1}\right|}
\end{array}\right) \text { in the case where } \alpha_{1} \neq 0
$$

and

$$
B_{\alpha}=\frac{\alpha_{2}}{\left|\alpha_{2}\right|}\left(\begin{array}{cc}
\frac{\alpha_{1} \alpha_{2}^{*}}{\left|\alpha_{2}\right|} & \left|\alpha_{2}\right| \\
\frac{\alpha_{3} \alpha_{2}^{*}}{\left|\alpha_{2}\right|} & \frac{\alpha_{4} \alpha_{2}^{*}}{\left|\alpha_{2}\right|}
\end{array}\right) \text { in the case where } \alpha_{2} \neq 0
$$

We note $a_{1} a_{4}-a_{2} a_{3}=\alpha_{1} \alpha_{4}^{*}-\alpha_{2} \alpha_{3}^{*}=1$ by (2.9). Therefore, we can complete our proof. 
Our main theorem in this paper is concerned with the classification of all the boundary conditions. To state the classification, we prepare another lemma. We denote by $S \mathbb{H}$ the Hamilton quaternion field with determinant one, i.e.,

$$
S \mathbb{H}:=\left\{\left.\left(\begin{array}{cc}
\gamma_{1} & -\gamma_{2}^{*} \\
\gamma_{2} & \gamma_{1}^{*}
\end{array}\right)\left|\gamma_{1}, \gamma_{2} \in \mathbb{C},\right| \gamma_{1}\right|^{2}+\left|\gamma_{2}\right|^{2}=1\right\} .
$$

The following lemma says that $U \in U(2)$ can be decomposed into the product of an element of $U(1)$ and an element of $S \mathbb{H}$. Although this lemma was already proved in [3. Proposition 4.3], we here give a simpler proof than that of [3, Proposition 4.3]:

Lemma 2.7. $U(2)=U(1) S \mathbb{H} \equiv\left\{\gamma_{3} A \mid \gamma_{3} \in U(1), A \in S \mathbb{H}\right\}$.

Proof. Since it is clear that $U(2) \supset U(1) S \mathbb{H}$, we show $U(2) \subset U(1) S \mathbb{H}$. Let $U=\left(\begin{array}{ll}u_{1} & u_{2} \\ u_{3} & u_{4}\end{array}\right)$ be an arbitrary matrix in $U(2)$. The equation $I_{\mathbb{C}^{2}}=U^{*} U$ implies

$$
\left|u_{1}\right|^{2}+\left|u_{3}\right|^{2}=1
$$

Similarly, the equation $I_{\mathbb{C}^{2}}=U U^{*}$ implies $\left|u_{1}\right|^{2}+\left|u_{2}\right|^{2}=1$. Comparing this with (2.12), we have

$$
\left|u_{2}\right|=\left|u_{3}\right| .
$$

In the same way, comparing the equation $\left|u_{3}\right|^{2}+\left|u_{4}\right|^{2}=1$ derived from $I_{\mathbb{C}^{2}}=U U^{*}$ with (2.12) leads to

$$
\left|u_{1}\right|=\left|u_{4}\right| .
$$

Here we introduce argument $\theta_{j}$ of $u_{j}$, i.e., $\theta_{j}:=\arg u_{j}$, and then $u_{j}=:\left|u_{j}\right| e^{i \theta_{j}}$, $j=1,2,3,4$. By using $I_{\mathbb{C}^{2}}=U U^{*},(2.13)$, and (2.14), we can reach

$$
\left|u_{1}\right|\left|u_{3}\right|\left(e^{i\left(\theta_{1}+\theta_{4}-\theta_{2}-\theta_{3}\right)}+1\right)=0 .
$$

In the case where $u_{3} \neq 0$, we set $\gamma_{1}, \gamma_{2}$, and $\gamma_{3}$ by $\gamma_{1}:=e^{-i\left(\theta_{2}+\theta_{3}+\pi\right) / 2} u_{1}, \gamma_{2}:=$ $e^{-i\left(\theta_{2}+\theta_{3}+\pi\right) / 2} u_{3}$, and $\gamma_{1}:=e^{i\left(\theta_{2}+\theta_{3}+\pi\right) / 2}$. Then, we have $\gamma_{1} \gamma_{3}=u_{1}$ and $\gamma_{2} \gamma_{3}=u_{3}$. By (2.13) we can compute $-\gamma_{2}^{*}$ as:

$$
-\gamma_{2}^{*}=-e^{i\left(\theta_{2}+\theta_{3}+\pi\right) / 2}\left|u_{3}\right| e^{-i \theta_{3}}=-e^{i\left(\theta_{2}-\theta_{3}+\pi\right) / 2}\left|u_{3}\right|=-e^{i\left(\pi-\theta_{2}-\theta_{3}\right) / 2} u_{2},
$$

which implies $-\gamma_{2}^{*} \gamma_{3}=-e^{i \pi} u_{2}=u_{2}$. Meanwhile, it follows from (2.15) that

$$
\left|u_{1}\right| e^{i\left(\theta_{1}+\theta_{4}\right)}=-\left|u_{1}\right| e^{i\left(\theta_{2}+\theta_{3}\right)} .
$$

Using this, we compute $\gamma_{1}^{*} \gamma_{3}$ as $\gamma_{1}^{*} \gamma_{3}=e^{i\left(\theta_{2}+\theta_{3}+\pi\right)}\left|u_{1}\right| e^{-i \theta_{1}}=-e^{i \pi}\left|u_{1}\right| e^{i \theta_{4}}=u_{4}$. Hence it follows that $U \in S \mathbb{H}$.

In the case $u_{3}=0$, we define $\gamma_{1}, \gamma_{2}$, and $\gamma_{3}$ by $\gamma_{1}:=e^{-i\left(\theta_{1}+\theta_{4}\right) / 2} u_{1}, \gamma_{2}:=0$, and $\gamma_{3}:=e^{i\left(\theta_{1}+\theta_{4}\right) / 2}$, and then, we have $\gamma_{1} \gamma_{3}=u_{1}, \gamma_{2} \gamma_{3}=0=u_{3}$. Since $u_{2}=0$ by (2.13), we have $-\gamma_{2}^{*}=0=u_{2}$. Finally, (2.14) brings us to the computation, $\gamma_{1}^{*} \gamma_{3}=e^{i\left(\theta_{1}+\theta_{4}\right)}\left|u_{1}\right| e^{-i \theta_{1}}=e^{i \theta_{4}}\left|u_{1}\right|=u_{4}$. Consequently, we obtain $U \in S \mathbb{H}$.

Proposition 2.3 based on von Neumann's theory says that all the self-adjoint extensions of the minimal Schrödinger operator $H_{0}$ are parameterized by $U \in U(2)$. Our assertion is that there are only two types of boundary conditions for all the selfadjoint extensions of $H_{0}$. They are represented by $(\overline{\mathrm{BC} \rho})$ and $(\overline{\mathrm{BC} \alpha})$. Therefore, the 
only thing we have to do for the classification of the boundary conditions is actually to construct the boundary condition $(\overline{\mathrm{BC} \rho})$ or $(\overline{\mathrm{BC} \alpha})$ from every $U \in U(2)$. In addition, Proposition 2.3 says that a diagonal $U \in U(2)$ leaves the set of the leftisland functions (resp. the right-island functions) invariant, on the other hand, a nondiagonal $U \in U(2)$ exchanges the left-island functions and the right-island functions. Theorem 2.8 shows that this situation is reflected in the boundary conditions.

THEOREM 2.8. For every self-adjoint extension $H_{U}, U \in U(2)$, of the minimal Schrödinger operator $H_{0}$, every boundary condition of the wave functions in $D\left(H_{U}\right)$ is constructed as in (a) or (b).

(a) Let $U \in U(2)$ be diagonal. Then, $U$ is represented as

$$
U=\left(\begin{array}{cc}
\gamma_{L} & 0 \\
0 & \gamma_{R}
\end{array}\right) \text { with }\left|\gamma_{L}\right|=\left|\gamma_{R}\right|=1
$$

The one-to-one correspondence between the self-adjoint extensions $H_{U}$ parameterized by diagonal matrices $U \in U(2)$ and the self-adjoint extensions $H_{\rho}$ parameterized by vectors $\rho \in \overline{\mathbb{R}}^{2}$ such that $D\left(H_{U}\right)=D\left(H_{\rho}\right)$ is given in the following:

(L1) For every $\gamma_{L} \neq-e^{i \sqrt{2} \Lambda}$, set $\theta_{L}$ as $\theta_{L}:=\arg \gamma_{L}$. Then, the component $\rho_{-} \in \mathbb{R}$ is given by

$$
\rho_{-}=-\frac{1}{\sqrt{2}}\left\{\tan \left(\frac{\theta_{L}}{2}-\frac{\Lambda}{\sqrt{2}}\right)-1\right\} .
$$

Conversely, for every $\rho_{-} \in \mathbb{R}$, the argument $\theta_{L}$ is determined by

$$
\theta_{L}=2 \arctan \left(-\sqrt{2} \rho_{-}+1\right)+\sqrt{2} \Lambda .
$$

(L2) The components $\gamma_{L}=-e^{i \sqrt{2} \Lambda}$ and $\rho_{-}=\infty$ correspond to each other.

(R1) For every $\gamma_{R} \neq-e^{i \sqrt{2} \Lambda}$, set $\theta_{R}$ as $\theta_{R}:=\arg \gamma_{R}$. Then, the component $\rho_{+} \in \mathbb{R}$ is given by

$$
\rho_{+}=\frac{1}{\sqrt{2}}\left\{\tan \left(\frac{\theta_{R}}{2}-\frac{\Lambda}{\sqrt{2}}\right)-1\right\} .
$$

Conversely, for every $\rho_{+} \in \mathbb{R}$, the argument $\theta_{R}$ is determined by

$$
\theta_{R}=2 \arctan \left(\sqrt{2} \rho_{+}+1\right)+\sqrt{2} \Lambda .
$$

(R2) The components $\gamma_{R}=-e^{i \sqrt{2} \Lambda}$ and $\rho_{+}=\infty$ correspond to each other.

(b) Let $U \in U(2)$ be non-diagonal. Then, $U$ is represented as

$$
U=\gamma_{3}\left(\begin{array}{cc}
\gamma_{1} & -\gamma_{2}^{*} \\
\gamma_{2} & \gamma_{1}^{*}
\end{array}\right) \text { with }\left|\gamma_{1}\right|^{2}+\left|\gamma_{2}\right|^{2}=\left|\gamma_{3}\right|=1 \text { and } \gamma_{2} \neq 0
$$

The one-to-one correspondence between the self-adjoint extensions $H_{U}$ parameterized by non-diagonal matrices $U \in U(2)$ and the self-adjoint extensions $H_{\alpha}$ parameterized by vectors $\alpha \in \mathbb{C}^{4}$ in the class (Class $\alpha$ ) such that $D\left(H_{U}\right)=D\left(H_{\alpha}\right)$ is given in the following: For every triple of components $\gamma_{1}, \gamma_{2}$, and $\gamma_{3}$, the vector $\alpha \in \mathbb{C}^{4}$ in the class (Class $\alpha$ ) is given by

$(\mathrm{TJF}-B)$

$$
\left\{\begin{array}{l}
\alpha_{1}=i \sqrt{2} \gamma_{2}^{-1}\left(\Re\left(e^{i \pi / 4} \gamma_{1}\right)+\Re\left(e^{i(\pi / 4-\sqrt{2} \Lambda)} \gamma_{3}\right)\right), \\
\alpha_{2}=-i \sqrt{2} \gamma_{2}^{-1}\left(\Re \gamma_{1}+\Re\left(e^{-i \sqrt{2} \Lambda} \gamma_{3}\right)\right), \\
\alpha_{3}=-i \sqrt{2} \gamma_{2}^{-1}\left(\Re \gamma_{1}+\Re\left(e^{i(\pi / 2-\sqrt{2} \Lambda)} \gamma_{3}\right)\right), \\
\alpha_{4}=i \sqrt{2} \gamma_{2}^{-1}\left(\Re\left(e^{-i \pi / 4} \gamma_{1}\right)+\Re\left(e^{i(\pi / 4-\sqrt{2} \Lambda)} \gamma_{3}\right)\right) .
\end{array}\right.
$$


Conversely, for every $\alpha \in \mathbb{C}^{4}$ satisfying (Class $\alpha$ ), the triple of the components $\gamma_{1}, \gamma_{2}$, and $\gamma_{3}$ is determined by

$$
\left\{\begin{array}{l}
\gamma_{1}=\Gamma_{0} e^{-i \theta}\left(e^{i \pi / 4} \alpha_{1}+\alpha_{2}+\alpha_{3}+e^{-i \pi / 4} \alpha_{4}\right), \\
\gamma_{2}=-i \sqrt{2} \Gamma_{0} e^{-i \theta}, \\
\gamma_{3}=-\Gamma_{0} e^{i(\sqrt{2} \Lambda-\theta)}\left(e^{-i \pi / 4} \alpha_{1}-i \alpha_{2}+\alpha_{3}+e^{-i \pi / 4} \alpha_{4}\right), \\
\Gamma_{0}=\left\{\left|e^{i \pi / 4} \alpha_{1}+\alpha_{2}+\alpha_{3}+e^{-i \pi / 4} \alpha_{4}\right|^{2}+2\right\}^{1 / 2} .
\end{array}\right.
$$

Theorems 2.4 and 2.8 show us the following correspondence: Every diagonal $U \in U(2)$ corresponds to the boundary condition $(\overline{\mathrm{BC} \rho})$, and every non-diagonal $U \in U(2)$ to the boundary condition $(\overline{\mathrm{BC} \alpha})$. In addition, Theorem 2.8 says that there is no boundary condition which makes a self-adjoint extension but the conditions $(\mathrm{BC} \rho)$ and $(\mathrm{BC} \alpha)$.

By Proposition 2.3. the wave functions belonging to the domain of every selfadjoint extension $H_{U}$ are represented as in (2.7). Since $\psi_{0}( \pm \Lambda)=0$ for $\psi_{0} \in D\left(H_{0}\right)=$ $A C_{0}^{2}\left(\overline{\Omega_{\Lambda}}\right)$, the unitary operator $U \in U(2)$ gives us the information about how the electron reflects at the boundary and how it passes through the junction. According to (2.8), the unitary operator $U$ maps the eigenfunction $L_{+}$living in the left island (resp. $R_{+}$living in the right island) to the eigenfunction $L_{-}$(resp. $R_{-}$) staying in the same island with the probability $\left|u_{11}\right|^{2}$ (resp. $\left|u_{22}\right|^{2}$ ) and the eigenfunction $R_{-}$ (resp. $L_{-}$) coming from the opposite island with the probability $\left|u_{12}\right|^{2}$ (resp. $\left|u_{21}\right|^{2}$ ).

Theorems 2.4 and 2.8 show how the information from $U \in U(2)$ reflects in the boundary conditions. The boundary condition $(\overline{\mathrm{BC} \rho})$ shows the solitariness:

$$
\text { (left island) } \begin{cases}\rho_{-} \psi(-\Lambda)=\psi^{\prime}(-\Lambda) & \text { if } \quad \rho_{-} \in \mathbb{R}, \\ \psi(-\Lambda)=0 & \text { if } \quad \rho_{-}=\infty\end{cases}
$$

and

$$
\text { (right island) }\left\{\begin{array}{lll}
\rho_{+} \psi(+\Lambda)=\psi^{\prime}(+\Lambda) & \text { if } & \rho_{+} \in \mathbb{R}, \\
\psi(+\Lambda)=0 & \text { if } & \rho_{+}=\infty
\end{array}\right.
$$

Both of the boundary conditions in the left island and the right one are independent of each other, which makes no interchange between the information of the individual wave functions living in the left island and right one. In addition, no extra phase factor $\theta$ appears with the form $e^{i \theta}$ in this boundary condition then. On the other hand, the boundary condition $(\overline{\mathrm{BC} \alpha})$ shows how the individual wave functions living in each island make interchange between each other at the boundary. Proposition 2.6 shows how a phase factor appears in the boundary condition:

$$
\left(\begin{array}{c}
\psi(+\Lambda) \\
\psi^{\prime}(+\Lambda)
\end{array}\right)=e^{i \theta}\left(\begin{array}{c}
a_{1} \psi(-\Lambda)+a_{2} \psi^{\prime}(-\Lambda) \\
a_{3} \psi(-\Lambda)+a_{4} \psi^{\prime}(-\Lambda)
\end{array}\right)
$$

for some $a_{j} \in \mathbb{R}, j=1, \cdots, 4$, with $a_{1} a_{4}-a_{2} a_{3}=1$. (TJF) with (TJF-B) and (TJF-U) is our tunnel-junction formula for the Schrödinger particle.

Using this tunnel-junction formula, we try to consider a possibility of a quantum device. That is, let us now consider a unit of a quantum device, consisting of a junction and two quantum wires as in Fig. 2.1 In this unit, the junction is for controlling the phase factor, and the wires for transporting the Schrödinger particle. In our argument 


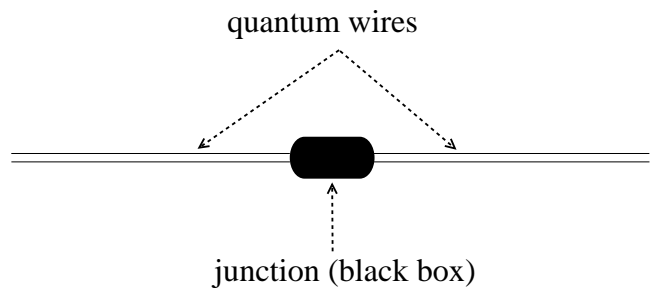

FIG. 2.1. The unit of our quantum device consists of the two quantum wires and the junction as the black box.

the two wires were respectively represented by the left island $\Omega_{\Lambda, L}$ and the right island $\Omega_{\Lambda, R}$, and the junction by the segment $[-\Lambda,+\Lambda]$. We regarded the junction as a black box to give mathematical, physical arbitrariness to the junction. As seen above, the self-adjointness of the Hamiltonian of the Schrödinger particle living in the two wires $\Omega_{\Lambda} \equiv \Omega_{\Lambda, L} \cup \Omega_{\Lambda, R}$ is mathematically determined by a boundary condition of the wave functions on which every self-adjoint extension acts. In real physics, actually, the boundary condition is uniquely determined by the quality and the shape of the boundary of a material of the wires. Thus, the wave functions have to satisfy the unit's own specific boundary condition to become the residents of the unit, otherwise the unit ejects them. We then consider the combination of different two units as in Fig 2.2. We set Unit0 with the boundary condition BC0 and Unit1 with the boundary condition $\mathrm{BC} 1$. We connect the two junctions with each other by a phase-controller. We employ $\theta_{0}$ for the phase factor in $\mathrm{BC} 0$ and $\theta_{1}\left(\neq \theta_{0}\right)$ for the phase factor in

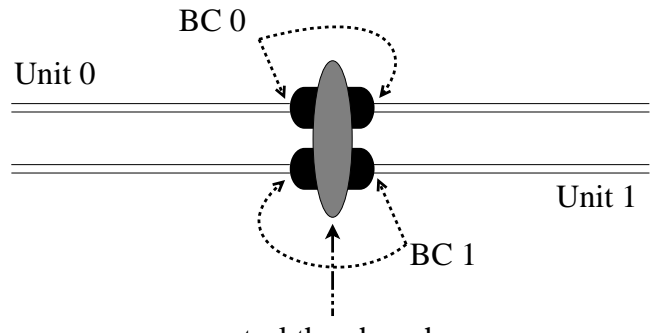

control the phase here

FIG. 2.2. The upper is Unit0 with the boundary condition BC0, and the lower is Unit1 with the boundary condition BC1. The ellipsoid body is a phase-controller.

$\mathrm{BC} 1$, respectively. If we succeed in the phase-shift gate operation from $\theta_{0}$ to $\theta_{1}$ at the phase-controller, we can make a phase-based switching device for electron as the Schrödinger particle. This suggests that we could use Unit0 and Unit1 as a qubit in the same way as the flying qubit [1] which is realized by the presence of an electron in either channel of the wires of an Aharonov-Bohm ring. Thus, our mathematical idea of the phase-based switching device may propose a possibility of the control over the transport of Schrödinger particle so that the transport through either channel of Unit0 and Unit1 makes another flying qubit by using phase factor.

\section{Proof of Theorems.}

3.1. Proof of Theorem 2.4, We here introduce the brief outline of the proof of Theorem 2.4 according to Theorem 1(ii) and Theorem 2(i). We will use the following lemma: 


\section{LEMMA 3.1.}

i) Let $a_{+}, a_{-}$be arbitrary complex numbers.

(i-1) For every $\rho=\left(\rho_{+}, \rho_{-}\right)$with $\left|\rho_{ \pm}\right|<\infty$, there is a wave function $\psi_{\rho} \in$ $D\left(H_{\rho}\right)$ such that $\psi_{\rho}( \pm \Lambda)=a_{ \pm}^{*}$.

(i-2) For every $\rho=\left(\rho_{+}, \rho_{-}\right)$with $\left|\rho_{+}\right|<\infty$ and $\rho_{-}=+\infty$, there is a wave function $\psi_{\rho} \in D\left(H_{\rho}\right)$ such that $\psi_{\rho}(+\Lambda)=a_{+}^{*}$ and $\psi_{\rho}^{\prime}(-\Lambda)=a_{-}^{*}$.

(i-3) For every $\rho=\left(\rho_{+}, \rho_{-}\right)$with $\rho_{+}=+\infty$ and $\left|\rho_{-}\right|<\infty$, there is a wave function $\psi_{\rho} \in D\left(H_{\rho}\right)$ such that $\psi_{\rho}(-\Lambda)=a_{-}^{*}$ and $\psi_{\rho}^{\prime}(+\Lambda)=a_{+}^{*}$.

(i-4) For every $\rho=\left(\rho_{+}, \rho_{-}\right)$with $\rho_{ \pm}=+\infty$, there is a wave function $\psi_{\rho} \in$ $D\left(H_{\rho}\right)$ such that $\psi_{\rho}^{\prime}( \pm \Lambda)=a_{ \pm}^{*}$.

ii) For arbitrary complex numbers $a_{1}, a_{2}$, there is a wave function $\psi_{\alpha} \in D\left(H_{\alpha}\right)$ such that $\psi_{\alpha}(-\Lambda)=a_{1}^{*}$ and $\psi_{\alpha}^{\prime}(-\Lambda) a_{2}^{*}$.

Proof. We denote by $\chi_{L}(x)$ and $\chi_{R}(x)$ the characteristic functions on $\overline{\Omega_{\Lambda, L}}=$ $(-\infty,-\Lambda]$ and $\overline{\Omega_{\Lambda, R}}=[+\Lambda,+\infty)$, respectively.

(i) Let $f, g$ be functions in $C_{0}^{\infty}(\mathbb{R})$ so that $f(0) \neq 0, f^{\prime}(0) / f(0)=1$, and $g(0)=0$, $g^{\prime}(0) \neq 0$. We define the function $\psi_{\rho}$ by

$$
\psi_{\rho}(x):=\frac{a_{-}^{*}}{f(0)} f\left(\rho_{-}(x+\Lambda)\right) \chi_{L}(x)+\frac{a_{+}^{*}}{f(0)} f\left(\rho_{+}(x-\Lambda)\right) \chi_{R}(x), \quad x \in \Omega_{\Lambda},
$$

for part (i-1),

$$
\psi_{\rho}(x):=\frac{a_{-}^{*}}{g^{\prime}(0)} g(x+\Lambda) \chi_{L}(x)+\frac{a_{+}^{*}}{f(0)} f\left(\rho_{+}(x-\Lambda)\right) \chi_{R}(x), \quad x \in \Omega_{\Lambda},
$$

for part (i-2),

$$
\psi_{\rho}(x):=\frac{a_{-}^{*}}{f(0)} f\left(\rho_{-}(x+\Lambda)\right) \chi_{L}(x)+\frac{a_{+}^{*}}{g^{\prime}(0)} g(x-\Lambda) \chi_{R}(x), \quad x \in \Omega_{\Lambda},
$$

for part (i-3),

$$
\psi_{\rho}(x):=\frac{a_{-}^{*}}{g^{\prime}(0)} g(x+\Lambda) \chi_{L}(x)+\frac{a_{+}^{*}}{g^{\prime}(0)} g(x-\Lambda) \chi_{R}(x), \quad x \in \Omega_{\Lambda},
$$

for part (i-4). Then, we obtain our desired function $\psi_{\rho}(x)$.

(ii) Let $f, g$ be functions in $C_{0}^{\infty}(\mathbb{R})$ so that $f(0) \neq 0, f^{\prime}(0)=0$, and $g(0)=0$, $g^{\prime}(0) \neq 0$. We define the function $\psi_{\rho}$ by

$$
\begin{aligned}
\psi_{\alpha}(x):=\left\{\frac{a_{1}^{*}}{f(0)} f(x+\Lambda)+\frac{a_{2}^{*}}{g^{\prime}(0)} g(x+\Lambda)\right\} \chi_{R}(x) \\
+\left\{\frac{b_{1}}{f(0)} f(x-\Lambda)+\frac{b_{2}}{g^{\prime}(0)} g(x-\Lambda)\right\} \chi_{R}(x),
\end{aligned}
$$

where $b_{1}=\alpha_{1} a_{1}^{*}+\alpha_{2} a_{2}^{*}$ and $b_{2}=\alpha_{3} a_{1}^{*}+\alpha_{4} a_{2}^{*}$. Then, the function $\psi_{\alpha}$ satisfies our desired condition.

It follows from integration by parts that

$$
\begin{aligned}
\left\langle H_{0}^{*} \psi \mid \phi\right\rangle_{L^{2}\left(\Omega_{\Lambda}\right)}-\left\langle\psi \mid H_{0}^{*} \phi\right\rangle_{L^{2}\left(\Omega_{\Lambda}\right)}= & \psi^{\prime}(+\Lambda)^{*} \phi(+\Lambda)-\psi(+\Lambda)^{*} \phi^{\prime}(+\Lambda) \\
& -\psi^{\prime}(-\Lambda)^{*} \phi(-\Lambda)+\psi(-\Lambda)^{*} \phi^{\prime}(-\Lambda)
\end{aligned}
$$

for every $\psi, \phi \in D\left(H_{0}^{*}\right)$. 
Since $H_{\sharp} \subset H_{0}^{*}$ for $\sharp=\rho, \alpha$, it is easy to check $H_{\sharp} \subset H_{\sharp}^{*}$ using (3.1). On the other hand, $H_{\sharp} \supset H_{\sharp}^{*}, \sharp=\rho, \alpha$, is proved as follows: By the general definition of adjoint operator, we have $0=\left\langle H_{\sharp} \psi_{\sharp} \mid \phi\right\rangle_{L^{2}\left(\Omega_{\Lambda}\right)}-\left\langle\psi_{\sharp} \mid H_{\sharp}^{*} \phi\right\rangle_{L^{2}\left(\Omega_{\Lambda}\right)}$ for every $\phi \in D\left(H_{\sharp}^{*}\right)$ and $\psi_{\sharp} \in D\left(H_{\sharp}\right)$ given in Lemma 3.1. The arbitrariness of $a_{+}$and $a_{-}$, or $a_{1}$ and $a_{2}$ implies $\phi \in D\left(H_{\sharp}\right)$, that is, $H_{\sharp}^{*} \subset H_{\sharp}$. This is our path to prove the self-adjointness of $H_{\sharp}$, i.e., $H_{\sharp}^{*}=H_{\sharp}$, employed in 2 .

For instance, we here show the proof of Theorem 2.4 (b) only. Since $H_{\alpha} \subset H_{0}^{*}$, we have

$$
\begin{aligned}
& \left\langle H_{\alpha} \psi \mid \phi\right\rangle_{L^{2}\left(\Omega_{\Lambda}\right)}-\left\langle\psi \mid H_{\alpha} \phi\right\rangle_{L^{2}\left(\Omega_{\Lambda}\right)} \\
= & \left(\alpha_{3} \psi(-\Lambda)+\alpha_{4} \psi^{\prime}(-\Lambda)\right)^{*}\left(\alpha_{1} \phi(-\Lambda)+\alpha_{2} \phi^{\prime}(-\Lambda)\right) \\
& -\left(\alpha_{1} \psi(-\Lambda)+\alpha_{2} \psi^{\prime}(-\Lambda)\right)^{*}\left(\alpha_{3} \phi(-\Lambda)+\alpha_{4} \phi^{\prime}(-\Lambda)\right) \\
& -\psi^{\prime}(-\Lambda)^{*} \phi(-\Lambda)+\psi(-\Lambda)^{*} \phi^{\prime}(-\Lambda) \\
= & \left(\alpha_{1} \alpha_{3}^{*}-\alpha_{1}^{*} \alpha_{3}\right) \psi(-\Lambda)^{*} \phi(-\Lambda)+\left(\alpha_{1} \alpha_{4}^{*}-\alpha_{2}^{*} \alpha_{3}-1\right) \psi^{\prime}(-\Lambda)^{*} \phi(-\Lambda) \\
& -\left(\alpha_{1}^{*} \alpha_{4}-\alpha_{2} \alpha_{3}^{*}-1\right) \psi(-\Lambda)^{*} \phi^{\prime}(-\Lambda)+\left(\alpha_{2} \alpha_{4}^{*}-\alpha_{2}^{*} \alpha_{4}\right) \psi^{\prime}(-\Lambda)^{*} \phi^{\prime}(-\Lambda) \\
= & 0
\end{aligned}
$$

for every $\psi, \phi \in D\left(H_{\alpha}\right)$ by using (3.1), (BC $\alpha$ ) and conditions of (Class $\left.\alpha\right)$. Hence it follows from this that $H_{\alpha}$ is symmetric, i,e, $H_{\alpha} \subset H_{\alpha}^{*}$.

Conversely, using the fact that $H_{\alpha} \subset H_{\alpha}^{*} \subset H_{0}^{*}$ along with the help of the general definition of adjoint operator and (3.1), for every $\phi \in D\left(H_{\alpha}^{*}\right)$ and $\psi_{\alpha} \in D\left(H_{\alpha}\right)$ given in Lemma 3.1(ii) we have

$$
\begin{aligned}
0= & \left\langle H_{\alpha} \psi_{\alpha} \mid \phi\right\rangle_{L^{2}\left(\Omega_{\Lambda}\right)}-\left\langle\psi_{\alpha} \mid H_{\alpha}^{*} \phi\right\rangle_{L^{2}\left(\Omega_{\Lambda}\right)} \\
= & \left(\alpha_{3} \psi_{\alpha}(-\Lambda)+\alpha_{4} \psi_{\alpha}^{\prime}(-\Lambda)\right)^{*} \phi(+\Lambda)-\left(\alpha_{1} \psi_{\alpha}(-\Lambda)+\alpha_{2} \psi_{\alpha}^{\prime}(-\Lambda)\right)^{*} \phi^{\prime}(+\Lambda) \\
& -\psi^{\prime}(-\Lambda)^{*} \phi(-\Lambda)+\psi(-\Lambda)^{*} \phi^{\prime}(-\Lambda) \\
= & a_{1}\left(\alpha_{3}^{*} \phi(+\Lambda)-\alpha_{1}^{*} \phi^{\prime}(+\Lambda)+\phi^{\prime}(-\Lambda)\right)+a_{2}\left(\alpha_{4}^{*} \phi(+\Lambda)-\alpha_{2}^{*} \phi^{\prime}(+\Lambda)-\phi(-\Lambda)\right) .
\end{aligned}
$$

So, the arbitrariness of $a_{1}$ and $a_{2}$ leads to

$$
\left(\begin{array}{c}
\phi(-\Lambda) \\
\phi^{\prime}(-\Lambda)
\end{array}\right)=\left(\begin{array}{cc}
\alpha_{4}^{*} & -\alpha_{2}^{*} \\
-\alpha_{3}^{*} & \alpha_{1}^{*}
\end{array}\right)\left(\begin{array}{c}
\phi(+\Lambda) \\
\phi^{\prime}(+\Lambda)
\end{array}\right)
$$

We here note that the conditions of (Class $\alpha$ ) leads to

$$
B_{\alpha}^{-1}=\left(\begin{array}{cc}
\alpha_{4}^{*} & -\alpha_{2}^{*} \\
-\alpha_{3}^{*} & \alpha_{1}^{*}
\end{array}\right)
$$

Thus, these two equations imply that $\phi \in D\left(H_{\alpha}\right)$. That is, $H_{\alpha}^{*} \subset H_{\alpha}$. Therefore, we have proved that $H_{\alpha}^{*}=H_{\alpha}$.

3.2. Proof of Theorem 2.8, Part (a) follows from [2, Theorem 1]. So, we prove part (b) only.

In this proof, we set $\eta:=e^{i \pi / 4}$ for simplicity. Let us give an arbitrary non-diagonal $U \in U(2)$. We know that $U \in U(2)$ has the representation in Lemma 2.7

By (2.7) we can write $\psi \in D\left(H_{U}\right)$ as

$$
\psi=\psi_{0}+c_{L} L_{+}+c_{R} R_{+}+c_{L} U L_{+}+c_{R} U R_{+},
$$


where $\psi \in D\left(H_{0}\right)$, and $c_{L}$ and $c_{R}$ run over $\mathbb{C}$ arbitrarily. Using this representation, (2.5) and (2.6) we can compute $\psi(+\Lambda), \psi^{\prime}(+\Lambda), \psi(-\Lambda)$, and $\psi^{\prime}(-\Lambda)$ as:

$$
\left\{\begin{array}{c}
\psi(+\Lambda)=-\gamma_{3} \gamma_{2}^{*} R_{+}(+\Lambda)^{*} c_{L}+\left\{R_{+}(+\Lambda)+\gamma_{3} \gamma_{1}^{*} R_{+}(+\Lambda)^{*}\right\} c_{R}, \\
\psi^{\prime}(+\Lambda)=e^{i \pi / 4} \gamma_{3} \gamma_{2}^{*} R_{+}(+\Lambda)^{*} c_{L} \\
-\left\{e^{-i \pi / 4} R_{+}(+\Lambda)+e^{i \pi / 4} \gamma_{3} \gamma_{1}^{*} R_{+}(+\Lambda)^{*}\right\} c_{R}, \\
\psi(-\Lambda)=\left\{R_{+}(+\Lambda)+\gamma_{3} \gamma_{1} R_{+}(+\Lambda)^{*}\right\} c_{L}+\gamma_{3} \gamma_{2} R_{+}(+\Lambda)^{*} c_{R}, \\
\psi^{\prime}(-\Lambda)=\left\{e^{-i \pi / 4} R_{+}(+\Lambda)+e^{i \pi / 4} \gamma_{3} \gamma_{1} R_{+}(+\Lambda)^{*}\right\} c_{L} \\
+e^{i \pi / 4} \gamma_{3} \gamma_{2} R_{+}(+\Lambda)^{*} c_{R} .
\end{array}\right.
$$

(3.2) says that

$$
\left(\begin{array}{c}
\psi(+\Lambda) \\
\psi^{\prime}(+\Lambda)
\end{array}\right)=A_{+}\left(\begin{array}{c}
c_{L} \\
c_{R}
\end{array}\right) \quad \text { and } \quad\left(\begin{array}{c}
\psi(-\Lambda) \\
\psi^{\prime}(-\Lambda)
\end{array}\right)=A_{-}\left(\begin{array}{c}
c_{L} \\
c_{R}
\end{array}\right)
$$

where

$$
A_{+}=\left(\begin{array}{cc}
-\gamma_{3} \gamma_{2}^{*} R_{+}(+\Lambda)^{*} & R_{+}(+\Lambda)+\gamma_{3} \gamma_{1}^{*} R_{+}(+\Lambda)^{*} \\
\eta \gamma_{3} \gamma_{2}^{*} R_{+}(+\Lambda)^{*} & -\left(\eta^{*} R_{+}(+\Lambda)+\eta \gamma_{3} \gamma_{1}^{*} R_{+}(+\Lambda)^{*}\right)
\end{array}\right)
$$

and

$$
A_{-}=\left(\begin{array}{cc}
R_{+}(+\Lambda)+\gamma_{3} \gamma_{1} R_{+}(+\Lambda)^{*} & \gamma_{3} \gamma_{2} R_{+}(+\Lambda)^{*} \\
\eta^{*} R_{+}(+\Lambda)+\gamma_{3} \gamma_{1} R_{+}(+\Lambda)^{*} & \gamma_{3} \gamma_{2} R_{+}(+\Lambda)^{*}
\end{array}\right)
$$

Since $\operatorname{det} A_{-}=i \sqrt{2}\left|R_{+}(+\Lambda)\right|^{2} \gamma_{3} \gamma_{2} \neq 0$, we know $A_{-}^{-1}$ exists. Thus, our desired representation TJF- $B$ of $\alpha_{1}, \alpha_{2}, \alpha_{3}$, and $\alpha_{4}$ by $\gamma_{1}, \gamma_{2}$, and $\gamma_{3}$ follows from the immediate computation of $B_{\alpha}=A_{+} A_{-}^{-1}$. Thus, every $\psi \in D\left(H_{U}\right)$ satisfies the boundary condition $(\mathrm{BC} \alpha)$. What we have to show next is that the vector $\alpha \in \mathbb{C}^{4}$ given by (TJF-B) is in the class (Class $\alpha$ ). It is obvious that our $\alpha_{1}, \alpha_{2}, \alpha_{3}$, and $\alpha_{4}$ satisfy (2.10). We can compute $\alpha_{1} \alpha_{4}^{*}-\alpha_{2} \alpha_{3}^{*}$ as follows:

$$
\begin{aligned}
\alpha_{1} \alpha_{4}^{*}-\alpha_{2} \alpha_{3}^{*}=\frac{2}{\left|\gamma_{2}\right|}\left[\left(\Re\left(\eta \gamma_{1}\right)+\Re\left(\eta e^{-i \sqrt{2} \Lambda} \gamma_{3}\right)\right)\left(\Re\left(\eta^{*} \gamma_{1}\right)+\Re\left(\eta e^{-i \sqrt{2} \Lambda} \gamma_{3}\right)\right)\right. \\
\left.-\left(\Re \gamma_{1}+\Re\left(e^{-i \sqrt{2} \Lambda} \gamma_{3}\right)\right)\left(\Re \gamma_{1}+\Re\left(\eta^{2} e^{-i \sqrt{2} \Lambda} \gamma_{3}\right)\right)\right] \\
=\frac{1}{2\left|\gamma_{2}\right|}\left[\left(\eta \gamma_{1}+\eta^{*} \gamma_{1}^{*}+\eta e^{-i \sqrt{2} \Lambda} \gamma_{3}+\eta^{*} e^{i \sqrt{2} \Lambda} \gamma_{3}^{*}\right)\right. \\
\quad \times\left(\eta^{*} \gamma_{1}+\eta \gamma_{1}^{*}+\eta e^{-i \sqrt{2} \Lambda} \gamma_{3}+\eta^{*} e^{i \sqrt{2} \Lambda} \gamma_{3}^{*}\right) \\
-\left(\gamma_{1}+\gamma_{1}^{*}+e^{-i \sqrt{2} \Lambda} \gamma_{3}+e^{i \sqrt{2} \Lambda} \gamma_{3}^{*}\right) \\
\left.\times\left(\gamma_{1}+\gamma_{1}^{*}+\eta^{2} e^{-i \sqrt{2} \Lambda} \gamma_{3}+\eta^{* 2} e^{i \sqrt{2} \Lambda} \gamma_{3}^{*}\right)\right] \\
=\frac{\left|\gamma_{3}\right|^{2}-\left|\gamma_{1}\right|^{2}}{\left|\gamma_{2}\right|^{2}}=1 .
\end{aligned}
$$

Thus, $\alpha_{1}, \alpha_{2}, \alpha_{3}$, and $\alpha_{4}$ given by (TJF-B) satisfy (2.9), and the vector $\alpha=$ $\left(\alpha_{1}, \alpha_{2}, \alpha_{3}, \alpha_{4}\right)$ is in the class (Class $\left.\alpha\right)$.

Therefore, we have constructed the boundary matrix $B_{\alpha}$ with the vector $\alpha \in \mathbb{C}^{4}$ in the class (Class $\alpha$ ) from every non-diagonal $U \in U(2)$. 
Conversely, let $\alpha_{1}, \alpha_{2}, \alpha_{3}$, and $\alpha_{4}$ be arbitrary complex numbers in the class (Class $\alpha$ ). It immediately follows from the definition of $\gamma_{1}, \gamma_{2}$, and $\Gamma_{0}$ in (TJF-UT that $\left|\gamma_{1}\right|^{2}+\left|\gamma_{2}\right|^{2}=1$. Using this equation together with the conditions of (Class $\alpha$ ) and the representation given in Proposition 2.6, we have

$$
\begin{aligned}
1 & =\left|\gamma_{1}\right|^{2}+\left|\gamma_{2}\right|^{2}=\Gamma_{0}^{2}\left\{\sum_{j=1}^{4}\left|a_{j}\right|^{2}+\sqrt{2}\left(a_{1} a_{2}+a_{1} a_{3}+a_{2} a_{4}+a_{3} a_{4}\right)+2 a_{1} a_{4}\right\} \\
& =\left|\gamma_{3}\right|^{2} .
\end{aligned}
$$

Here we note $\gamma_{2} \neq 0$ by its definition. Thus, TJF-U gives us the unitary operator $U$ with the representation:

$$
U=\gamma_{3}\left(\begin{array}{cc}
\gamma_{1} & -\gamma_{2}^{*} \\
\gamma_{2} & \gamma_{1}^{*}
\end{array}\right) \in U(1) S \mathbb{H}=U(2) .
$$

We show from now on that the above $U$ satisfies $D\left(H_{U}\right)=D\left(H_{\alpha}\right)$.

For arbitrarily given $U \in U(2)$, insert $\psi(+\Lambda), \psi^{\prime}(+\Lambda), \psi(-\Lambda)$, and $\psi^{\prime}(-\Lambda)$ with the representation (3.2) into the boundary conditions,

$$
\left\{\begin{array}{l}
\psi(+\Lambda)=\alpha_{1} \psi(-\Lambda)+\alpha_{2} \psi^{\prime}(-\Lambda), \\
\psi^{\prime}(+\Lambda)=\alpha_{3} \psi(-\Lambda)+\alpha_{4} \psi^{\prime}(-\Lambda) .
\end{array}\right.
$$

Then, since the coefficients $c_{L}$ and $c_{R}$ in $D\left(H_{U}\right)$ are arbitrary and $\gamma_{3}^{-1}=\gamma_{3}^{*}$, we can show that the condition $D\left(H_{U}\right)=D\left(H_{\alpha}\right)$ is equivalent to the system of the following system of equations:

$$
\begin{aligned}
& \left(\alpha_{1}+e^{i \pi / 4} \alpha_{2}\right) \gamma_{2}-\gamma_{1}^{*}=e^{i \sqrt{2} \Lambda} \gamma_{3}^{*}, \\
& \gamma_{2}^{*}+\left(\alpha_{1}+e^{i \pi / 4} \alpha_{2}\right) \gamma_{1}=-\left(\alpha_{1}+e^{-i \pi / 4} \alpha_{2}\right) e^{i \sqrt{2} \Lambda} \gamma_{3}^{*}, \\
& e^{i \pi / 4} \gamma_{2}^{*}-\left(\alpha_{3}+e^{i \pi / 4} \alpha_{4}\right) \gamma_{1}=\left(\alpha_{3}+e^{-i \pi / 4} \alpha_{4}\right) e^{i \sqrt{2} \Lambda} \gamma_{3}^{*}, \\
& \left(\alpha_{3}+e^{i \pi / 4} \alpha_{4}\right) \gamma_{2}+e^{i \pi / 4} \gamma_{1}^{*}=-e^{i(\sqrt{2} \Lambda-\pi / 4)} \gamma_{3}^{*} .
\end{aligned}
$$

Thus, we now show that our $U$ given by (TJF- $U$ ) satisfies the system of equations: Using the representation in Proposition 2.6, it is easy to check that our $\gamma_{1}, \gamma_{2}$, and $\gamma_{3}$ given by (TJF-U) satisfy (3.3) and (3.6) in the following.

$$
\begin{aligned}
& \left(\alpha_{1}+\eta \alpha_{2}\right) \gamma_{2}-\gamma_{1}^{*}=-\Gamma_{0}\left(\eta a_{1}+i a_{2}+a_{3}+\eta a_{4}\right)=e^{i \sqrt{2} \Lambda} \gamma_{3}^{*}, \\
& \left(\alpha_{3}+\eta \alpha_{4}\right) \gamma_{2}+\eta \gamma_{1}^{*}=\Gamma_{0}\left(a_{1}+\eta a_{2}+\eta^{*} a_{3}+a_{4}\right)=-\eta^{*} e^{i \sqrt{2} \Lambda} \gamma_{3}^{*} .
\end{aligned}
$$

We recall $a_{1} a_{4}-a_{2} a_{3}=1$ by Proposition 2.6. This equation leads to

$$
\begin{aligned}
& \eta a_{1} a_{4}+i \eta^{*} a_{2}^{2}+\eta^{*} a_{2} a_{3}=\eta^{*} a_{1} a_{4}+\eta a_{2}^{2}+\eta a_{2} a_{3}+i \sqrt{2}, \\
& a_{1} a_{4}+i a_{2} a_{3}=i a_{1} a_{4}+a_{2} a_{3}+1-i .
\end{aligned}
$$

We can show that our $\gamma_{1}, \gamma_{2}$, and $\gamma_{3}$ satisfy (3.4) as

$$
\begin{gathered}
\gamma_{2}^{*}+\left(\alpha_{1}+\eta \alpha_{2}\right) \gamma_{1}=e^{i \theta} \Gamma_{0}\left\{\eta a_{1}^{2}+(1+i) a_{1} a_{2}+a_{1} a_{3}+\eta^{*} a_{1} a_{4}\right. \\
\left.+\eta a_{2}^{2}+\eta a_{2} a_{3}+a_{2} a_{4}+i \sqrt{2}\right\} \\
=-\left(\alpha_{1}+\eta^{*} \alpha_{3}\right) e^{i \sqrt{2} \Lambda} \gamma_{3}^{*}
\end{gathered}
$$


with the help of (3.7), and they satisfy (3.4) as

$$
\begin{aligned}
\eta \gamma_{2}^{*}-\left(\alpha_{3}+\eta \alpha_{4}\right) \gamma_{1}= & -e^{i \theta} \Gamma_{0}\left\{\eta a_{1} a_{3}+i a_{1} a_{4}+a_{2} a_{3}+\eta a_{2} a_{4}\right. \\
& \left.+a_{3}^{2}+\sqrt{2} a_{3} a_{4}+a_{4}^{2}+1-i\right\} \\
= & \left(\alpha_{3}+\eta^{*} \alpha_{4}\right) e^{i \sqrt{2} \Lambda} \gamma_{3}^{*}
\end{aligned}
$$

with the help of (3.8).

Therefore, the unitary operator $U$ made from our $\gamma_{1}, \gamma_{2}$, and $\gamma_{3}$ satisfies the equation $D\left(H_{U}\right)=D\left(H_{\alpha}\right)$, and we can complete the proof of our theorem.

4. Conclusion. We have completely characterized the boundary conditions for all the self-adjoint extensions of the minimal Schrödinger operator. We then found a tunnel-junction formula concerning the phase factor. In this formula we can find the factor $\pm \sqrt{2} \Lambda$ which depends on the length of the junction. We have not yet clarified the physical reason why this factor appears. Compared with the results in 3] and this paper, we realize that such a factor concerning the parameter $\Lambda$ appears for the Schrödinger particle, but it does not for the Dirac particle [3. That is, the Schrödinger particle seems to feel the distance $2 \Lambda$, though the Dirac particle does not. We conjecture that this situation physically depends on the speed of the particle.

Acknowledgments. One of the authors (M.H.) acknowledges the financial support from JSPS, Grant-in-Aid for Scientific Research (C) 23540204. He also expresses special thanks to Kae Nemoto and Yutaka Shikano for the useful discussions with them.

\section{REFERENCES}

[1] M. Yamamoto, S. Takada, C. Bäuerle, K. Watanabe, A. D. Wieck, and S. Tarucha, Electrical control of a solid-state flying qubit, Nature Nanotechnology, 7 (2012), pp. 247251.

[2] Y. Furuhashi, M. Hirokawa, K. Nakahara, and Y. Shikano, Role of Phase Factor in Boundary Condition of One-Dimensional Junction, J. Phys. A: Math. Theo., 43 (2010), 354010.

[3] M. Hirokawa and T. Kosaka, A Mathematical Aspect of A Tunnel-Junction Device for Spintronic Qubit, arXiv:1306.2688

[4] Y. Shikano And M. Hirokawa, Boundary Conditions in One-dimensional Tunneling Junction, J. Phys.: Conference Series, 302 (2011), 0124044.

[5] M. Reed and B. Simon, Methods of Modern Mathematical Physics II. Fourier Analysis, SelfAdjointness, Academic Press, San Diego, 1975.

[6] J. Weidmann, Linear Operators in Hilbert Spaces, Springer-Verlag, New York, 1980. 